\title{
Breath Alcohol Test Device
}

National Cancer Institute

\section{Source}

National Cancer Institute. Breath Alcohol Test Device. NCI Thesaurus. Code C99266.

A device that approximates a blood alcohol level from the components of an expired breath. 